\title{
Über eine Heptose im menschlichen Urin.
}

\author{
Von
}

Dr. F. Rosenberger.

(Aus dem physiologischen Institut der Universität Heidelberg.)

(Der Redaktion zugegangen am 31. Juli 1906.)

Im folgenden gebe ich Beobachtungen wieder, die ich am Urin einer Patientin im Laufe des letzten Jahres gemacht habe, über deren Uratausscheidung und sonstige klinische Verhältnisse, da die Krankengeschichte sehr interessant ist, an anderer Stelle ${ }^{1}$ ) berichtet werden wird.

So lange die Kranke auf der medizinischen Abteilung des Juliusspitals zu Würzburg lag, gelang es mir, in ihrem Urin durch Ausziehen mit Methyl- resp. Äthylalkohol und Fällen mit Äther eine optisch inaktive, eine rechtsdrehende und eine dritte Substanz nach Möglichkeit zu isolieren, die im Laufe der Untersuchungen zuerst schwach links drehte, dann aber aus völlig unbekannten Gründen optisch inaktiv wurde.

Die rechtsdrehende Substanz war gärfähig mit reiner Bierhefe. Die beiden andern waren es nicht; auch der Versuch, sie mit Salzsäure zu invertieren, gab ein völlig negatives Resultat. Alle drei Substanzen reduzierten Kupferlösung und beim Erwärmen ammoniakalische Silberlösung. Osazone konnte ich nur aus der rechtsdrehenden Lösung gewinnen, in den beiden andern bildete sich nur eine gelbe, wolkige Trübung, in der sich unter dem Mikroskop vereinzelte Krystalle nachweisen ließen. Ich betone ausdrücklich, daß ich zur Herstellung der Osazone nur 45 Minuten lang das Gemisch von Lösung, Phenylhydrazin und Essigsäure auf dem Wasserbad erhitzte; es ist wichtig, diesen Punkt im Auge zu behalten! Durch Bleiacetat (10\% ige neutrale Lösung) wurde keiner der drei Körper gefällt. Die Seliw anoffsche Reaktion gab nur die linksdrehende Lösung sofort nach Kochen mit Salzsäure; die beiden anderen erst nach langem Kochen. Das Osazon der rechtsdrehenden Substanz war frischbereitet gelb, wurde aber nach einigem Liegen

1) Deutsches Archiv für klinische Medizin und Zeitschrift für Biologie. 
braun. Ich versuchte des öfteren, wenn der Urin der Kranken reduzierende Eigenschaften hatte, aus demselben direkt Osazone herzustellen, hatte aber sehr oft keinen Erfolg, es schied sich jeweils ein schwarzbraunes Öl ab. Das Erhitzen geschah in diesen Fällen jedesmal nur 45 Minuten lang.

Von den an verschiedenen Tagen erhaltenen Osazonen schmolzen die meisten bei $203-204^{\circ}$, ich erhielt aber auch solche, die hartnäckig bei $196^{\circ}$ schmolzen, nachdem sie sich bei $188-190^{\circ}$ gebräunt hatten. Umkrystallisieren zeigte sich trotz häufigen Wiederholens als nutzlos, d. h. ohne Einfluß auf den Schmelzpunkt; einmal wurden derartige Osazone mit $96 \%$ igem Alkohol aufgeschwemmt, im Filtrat fanden sich dann Krystalle, die bei $203-204^{\circ}$ schmolzen und diesen Schmelzpunkt auch nach dem Umkrystallisieren hielten. Eine Drehung der Polarisatjonsebene konnte ich an Lösungen der Osazone in Eisessig nie beobachten. Auch Osazone vom Schmelzpunkt $206^{\circ}$ erhielt ich.

Ich halte es nicht für unwichtig, darauf hinzuweisen, daß der Urin sehr oft dadurch auffiel, daß er zwar stark reduzierte, dabei aber kein Kupferlösungsvermögen besaß, auch wurde gelegentlich in Abwesenheit von Eiweiß bei negativer Eisenchloridreaktion eine schwache Linksdrehung (am 21./22. Nov. 1905 sogar entsprechend der Rechtsdrehung einer 0,3\% igen Traubenzuckerlösung) beobachtet.

Das einzige, was aus dem bisherigen Ergebnis gefolgert werden konnte, war, daß hier bei einem klinisch hochinteressanten Fall im Urin offenbar verschiedene Zuckerarten ausgeschieden wurden.

Als ich die Stelle als Assistent der medizinischen Abteilung des Juliusspitals in Würzburg aufgegeben hatte, behielt ich daher die Kranke, die ihren Wohnsitz in W. hat, im Auge und setzte die Untersuchungen am physiologischen Institut in Heidelberg fort. Am 26. März 1906 erhielt ich $150 \mathrm{ccm}$ Urin, welcher ein geringes Kupferlösungsvermögen hatte, nach längerem Erhitzen sehr stark reduzierte, rechts drehte. Einen Gärversuch habe ich mit diesem Urin nicht gemacht, dagegen muß ich bemerken, daß der in diesen Tagen gelassene Harn bei starkem Reduktionsvermögen gelegentlich nicht gor (was ich geneigt 
war, sogar auf Versuchsfehler zurückzuführen). Die $150 \mathrm{ccm}$ Urin verarbeitete ich auf Osazone und ließ sie länger als früher auf dem kochenden Wasserbad. Die Ausbeute war eine sehr gute.

Ich versuchte die Osazone nach ihrer Löslichkeit in Alkohol von einander zu trennen. Zunächst wurden sie in heißem Alkohol gelöst, dann ein Teil des Alkohols abgedampft, durch Zusatz kalten Wassers wurden einige Osazone ausgefällt, filtriert, weiter eingeengt usf. Das Ausfällen mit Wasser aus heißem Alkohol halte ich, namentlich da, wo es sich um frisch aus Urin gewonnene Osazone handelt, schon deshalb für praktisch, weil dadurch Verunreinigungen mit Phenylsemicarbacid vermieden werden. Die schwerstlöslichen Krystalle zeigten sofort den höchsten Schmelzpunkt $\left(195-196^{\circ}\right)$, die anderen erreichten deņ̧elben größtenteils, nachdem sie vom Gesichtspunkt der Schwerlöslichkeit aus mehrfach in der angegebenen Weise umkrystallisiert waren. Die Körper waren gelb oder rotgelb und hatten die charakteristische Eigenschaft, vor dem Schmelzen zu schrumpfen und sich tief zu bräunen. Gerade diese Verfärbung, die mir schon in Würzburg einige Male aufgefallen war, trat desto genauer bei $190^{\circ}$ ein, je reiner die Substanz war.

Emil Fischer( $\left.{ }^{1}\right)$ hat im Jahr 1892 die Synthese einiger Heptosen angegeben, deren Osazon bei $195^{\circ}$ nach vorangehender Braunfärbung $\left(190^{\circ}\right)$ schmilzt und sich sehr schwer in siedendem absoluten Alkohol löst.

Müntz und Marcano,(2) Maquenne( $\left.{ }^{3}\right)$ haben unter dem Namen "Perseit», Bourquelot $\left({ }^{4}\right)$ unter dem Namen "Volemit» siebenwertige Alkohole - d-Mannoheptose bezw. Volemose beschrieben, die sie in Persea gratissima und Lactuarius volemus gefunden hatten. Den Volemit haben des weiteren Bougault und Allard(5) im Trockenrückstand einiger Primulaceen nachgewiesen.

Trotzdem hielt ich mich noch nicht für berechtigt, die fragliche Substanz als Heptose anzusprechen, sondern glaubte um so eher nur ein Gemisch verschiedener Körper (Hexosen und Biosen) vor mir zu haben, als ich aus größeren Mengen (4 l) Urin, die ich mir unter Toluolzusatz hatte schicken lassen, Körper von verschiedenem "Verhalten darstellte. 
Bei den weiteren Versuchen ging ich darauf aus, den fraglichen Zucker rein darzustellen, und verfuhr nach dem im folgenden gegebenen Verfahren, dessen Erfolg zugleich einige Eigenschaften des Körpers feststellen ließ, und eine Vermutung in mir weckte, der ich am Schluß Raum geben werde.

$4000 \mathrm{ccm}$ Urin wurden mit 10\% iger neutraler Bleiacetatlösung, das mit $\mathrm{H}_{2} \mathrm{~S}$ entbleite Filtrat mit Phosphorwolframsäure gefällt, die überschüssige Phosphorwolframsäure mit Barythydrat aus dem Filtrat entfernt, der durch Eindampfen im Vakuum erhaltene Trockenrückstand in Methylalkohol bei $0^{0} \mathrm{G}$. mit methylalkoholischer Barytlösung ausgefällt. Das Filtrat dieser Fällung mit $\mathrm{H}_{2} \mathrm{SO}_{4}$ abgestumpft und mit $\mathrm{H}_{2} \mathrm{CO}_{3}$ vom letzten Rest Baryt befreit, war eine braune Flüssigkeit, die stark bei alkalischer Reaktion Kupfer reduzierte, ohne es vorher zu lösen, bei saurer (Barfoed) dagegen nicht. Sie war optisch inaktiv; die reduzierende Substanz ging auf Zusatz von basisch-essigsaurem Blei sowohl in den Niederschlag als auch in das Filtrat. Die Osazone drehten in Pyridin gelöst die Polarisationsebene auch in großer Menge nicht. Schleimsäure entstand beim längeren Erhitzen mit Salpetersäure nicht, auch konnte ich keine Zuckersäure nach entsprechender Behandlung nachweisen. Die Gärprobe war negativ mit Saccharomyces cerevisiae, Bact. coli commune, Bact. acidi lactici, Oidium lactis. Diese Stämme erhielt ich durch die Güte des Herrn Prof. Dr. Neumann aus dem Heidelberger hygienischen Institut, wofür ich ihm meinen verbindlichsten Dank sage.

Eine Änderung im chemischen, optischen und biologischen Verhalten trat auch nach längerem Kochen mit verdünnter Salzsäure oder nach Speichelzusatz bei der in Frage stehenden Substanz nie ein. Durch Kochen mit starker Salzsäure erhielt ich sehr viel Huminsubstanz unter Kohlensäureabscheidung, der Geruch war furfurolartig. Mit dünner Natronlauge färbte sich die Lösung beim Kochen gelb. Das Kupfer wurde von ihr mit roter Farbe reduziert.

Die Elementaranalyse der am 27. März 1906 hergestellten Osazone ergab $(0,2613 \mathrm{~g}$ Substanz): 
Gefunden: Berechnet für Heptosazon

Für Hexosazon

$\begin{array}{rcr} & \left(\mathrm{C}_{18} \mathrm{H}_{24} \mathrm{O}_{5} \mathrm{~N}_{4}\right): & \left(\mathrm{C}_{18} \mathrm{H}_{22} \mathrm{O}_{4} \mathrm{~N}_{4}\right): \\ 14,8 \% \mathrm{~N} & 14,47 \% \mathrm{~N} & 15,6 \% \mathrm{~N} \\ & \text { Verwendet: } 0,047 \mathrm{~g} \text { S Substanz: } & \\ 58,89 \% \mathrm{C} & 58,8 \% \mathrm{C} & 60,3 \% \mathrm{C} \\ 6,19 \% \mathrm{H} & 6,18 \% \mathrm{H} & 6,14 \% \mathrm{H}\end{array}$

Die gefundenen Werte sind so weit von den für das Hexosazon berechneten entfernt und liegen dem des Heptosazons so nahe, daß sie zugunsten meiner Annahme sprechen. Leider konnte ich keine weitere Kohlenwasserstoffanalyse mit mehr Substanz machen.

Eine Sendung vom 12. Juni 1906 hatte zu wenig reduzierende Substanz enthalten, wie denn schon im Winter 1905/06 der Zuckergehalt ohne erkenntliche Ursache plötzlichen großen Schwankungen unterworfen gewesen war. Am 11. Juli 1906 wurden mir 6 Flaschen $=4200 \mathrm{ccm}$ Urin geschickt. 2 Flaschen davon enthielten keinen Zucker, der Inhalt von 2 anderen gab Osazone vom Schmelzpunkt $203^{\circ}$ bezw. 198-199 ${ }^{\circ}$, der der beiden übrigen solche, die sich nach vorangehender Bräunung bei $195^{\circ}$ verflüssigten. Der letztere Urin wurde verarbeitet.

Analyse der Osazone (0,1085 g Substanz):

Gefunden:

$14,542 \% \mathrm{~N}$
Berechnet für Heptosazon:

$14,47 \% \mathrm{~N}$

Zur weiteren Untersuchung stellte ich das Oson und das Benzoylat her. Das Oson wurde aus etwas über $3 \mathrm{~g}$ der am 27. März 1906 und aus etwa $2 \mathrm{~g}$ der am 11. Juli hergestellten Osazone gewonnen. Der nach gemeinsamem Umkrystallisieren zuvor festgestellte Schmelzpunkt - $195^{\circ} \mathrm{C}$. - dieser Osazone war durch die Mischung unverändert geblieben. Er fand sich später wieder an den Krystallen, die sich bei sehr gelindem Erwärmen fast augenblicklich bildeten, als zum Oson Phenylhydrazin und Essigsäure gefügt wurden. Gleiche Osazone lieferte der durch Reduktion mit Zinkstaub und Eisessig aus den Osonen entstandene Zucker. Dieser letztere war nicht gärfähig mit Saccharomyces cerev. und Bact. ac. lact., reduzierte Kupferhydroxyd, ohne es vorher zu lösen. Optisch erwies er sich inaktiv, allerdings war die untersuchte Menge sehr gering. Vom Ausgangsmaterial unterschied er sich dadurch, daß seine Lösung, 
mit Orcin und Salzsäure gekocht, sich rötete und spektroskopisch das in Amylalkohol mit roter Farbe gelöste Pigment das Violett völlig auslöschte. Die Ausgangslösung färbte sich bei diesem Verfahren braun mit einem Stich, ins Rötliche, der Amylalkohol wurde schwarzbraun, im Spektrum war Grün teilweise, alles Übrige nach rechts völlig erloschen.

Durch Schütteln mit Benzoylchlorid und Kalilauge entstanden Benzoylate, die aus warmem Alkohol durch Schütteln mit Wasser (1,0 Benzoylat : 1000,0 Wasser) umkrystallisiert, dann mit überschüssiger Salzsäure (1,1 spez. Gew.) in Bombenröhren eingeschlossen und durch 12 Stunden auf $100^{\circ}$ unter häufigem Schütteln erhitzt wurden. Dieses Verfahren hat sich nach F. Müller( $\left.{ }^{6}\right)$ zur Darstellung des Glykosamins bewährt, und deshalb wandte ich es sofort an, da mir keine Zeit mehr blieb, eine andere Art der Verseifung auszuprobieren. Die Heptose scheint aber sehr empfindlich zu sein, da sich eine sehr große Menge Kohle bildete. Was nach der angegebenen Zeit unverseift, war, wurde nochmals eingeschmolzen und weitere 12 Stunden erhitzt. Der Druck in den Röhren war äußerst gering, ich erhielt eine stark reduzierende, optisch inaktive, braune Flüssigkeit, der mit Äther die Benzoesäure entzogen wurde. Die Salzsäure fällte ich, nachdem ein Vorversuch bei Zimmertemperatur die Unschädlichkeit des Verfahrens erwiesen hatte, mit Bleicarbonat bei $0^{\circ}$, den Rest, nach Einengen bei niederer Temperatur im Vakuum, behandelte ich abgekühlt auf $0^{\circ}$ mit Silbercarbonat ebenfalls in der Reibschale. Diese Methode war mir von der Herstellung der Osone her als schonend bekannt. Die Ausbeute war eine sehr geringe. Ich erhielt einen hellbraunen Sirup, der die schon angegebenen Eigenschaften der aus der Methylalkoholfällung im Filtrat erhaltenen Substanz hatte und ganz schwach süß, aber dann fad bittersalzig schmeckte, wenn ich einen Tropfen auf die Mitte der Zunge legte und im Speichel ohne besondere Bewegung der Zunge diffundieren ließ. Die Osazone auch dieses Körpers bräunten sich bei $190^{\circ}$ präzis und schmolzen bei $195^{\circ}$.

Den Schmelzpunkt habe ich des öfteren im Laufe der Untersuchungen mit verschiedenen Thermometern geprüft und 
stets bei $195-196^{\circ}$ gefunden, wenn ich bis $175^{\circ}$ sehr stark und rasch, von da an ruhig erhitzte, sodaß die Bestimmung jeweils 5-6 Minuten Zeit in Anspruch nahm.

Aus dem Gesagten geht, glaube ich, hervor, daß die von mir behandelte Substanz eine einheitliche, nach ihrer Bearbeitung auch eine reine war, und aus den Elementaranalysen wie dem Schmelzpunkt der Osazone, daß es sich um eine Heptose handelte.

0 . Simon $\left({ }^{7}\right)$ gelang die Darstellung einer Albumose aus der Schweineleber, aus der sich ein Kohlehydrat abspalten ließ, dessen Osazon «um $190^{\circ} \mathrm{C}$.» schmolz. Es gelang mir nicht, dasselbe $\mathrm{zu}$ gewinnen; daß es eine Heptose ist, halte ich trotz seiner starken Gärfähigkeit nicht für ausgeschlossen, denn die Zucker mit sieben Kohlenstoffatomen scheinen doch nicht so selten $\mathrm{zu}$ sein, als man geneigt sein möchte zu glauben, und dieser Umstand erhebt daher meinen Befund über das Niveau eines bloßen Kuriosums. 1887 beschrieb Leo $\left(^{8}\right)$ einen Körper, der nach den äußerst mühsamen Untersuchungen dieses Forschers die Strukturformel: $\mathrm{C}_{n} \mathrm{H}_{2 \mathrm{n}} \mathrm{O}_{\mathrm{n}}$ hat, dessen Osazone er nicht erhielt, da er nur kurz erwärmte. Die Ähnlichkeit dieser «Laiose» mit der von mir gefundenen Heptose ist eine außerordentlich weitgehende, auch in dem Punkt deckt sich Leos Befund mit dem meinen, daß im vergorenen Urin die Linksdrehung fehlt. Diese Tatsache läßt sich zwanglos mit den modernen Anschauungen über das Wesen der Gärung in Einklang bringen, die die Zugänglichkeit der verschiedenen Zucker den Spaltpilzen gegenüber vom osmotischen Druck der Lösung abhängig machen (Gegenwart von Kochsalz in verschiedenen Mengen ermöglichte in meinem Fall bei einigen Versuchen die Gärung der reinen Heptose nicht).

Auch die noch mehr oder minder problematischen 0xydasen mögen durch ihre Gegenwart im Urin den Hefepilzen das Spiel erleichtert haben.

Von den Pentosen ist bekannt, daß sie zwar an sich schwer, bei Gegenwart anderer Zucker aber mitunter glatt vergoren werden.

Das von Leo bestimmte Drehungsvermögen der Laiose $=-25,41^{\circ}$ entspricht dem der Glukoheptose $=-25^{\circ}$. Auch 
dieser Umstand spricht für die Annahme, daß die Laiose eine Heptose ist. Solange ich in Heidelberg war, fand ich nie eine linksdrehende Substanz, dagegen drehte der Urin der Patientin in Würzburg mehrfach links; es ist also möglich, daß sie damals optisch aktive, später racemische Heptose ausschied.

Die Methylhexosen sind in ihrem Verhalten von den Heptosen wesentlich unterschieden und liefern Schleimsäure beim Erhitzen mit Salpetersäure.

Der Nachweis einer Heptose beim Menschen setzt den Arzt in die Lage, sich im einzelnen Diabetesfall eingehender als bisher mit der Natur des vorliegenden Zuckers zu befassen, zumal das l-Mannoheptosazon den Schmelzpunkt $203^{\circ}$ hat.

Es ist ein echt menschlicher Zug, daß nur das in erster Linie erforschenswert erscheint, was unmittelbar mit dem Menschen zu tun hat. Bisher waren die Heptosen mehr Gegenstand trockener, wissenschaftlicher Experimente, eine praktische Bedeutung wurde ihnen nicht zuerkannt, möglich daß auch von ihnen in einiger Zeit gesagt werden kann, was Debove $\left(^{9}\right)$ über den Diabetes bei Eheleuten gesagt haben soll: «Les faits sur lesquels notre attention n'a pas été attirée paraissent toujours exeptionnels; ils deviennent fréquents dès qu'on les recherche».

Herrn Prof. Dr. Kossel spreche ich für die gütige Aufnahme in seinem Laboratorium, die Erlaubnis der Bearbeitung des Themas und zahlreiche, freundliche Anregungen und Ratschläge hochachtungsvollst meinen Dank aus.

\section{Literatur :}

1. Justus Liebigs Annalen der Chemie, Bd. CGLXX, H. 1 u. 2, S. 64.

2. Annales de Chimie et de physique, vol. VI, 3, p. 279.

3. Comptes rendus de l'académie des sciences 106, 1235 u. 107, 583.

4. Chemikerzeitung 15, R. 190.

5. Comptes rendus de l'académie des sciences 135, 796.

6. Friedrich Müller, Zeitschrift f. Biologie, Bd. XLII.

7. Arch. f. exp. Path. u. Pharm., Bd. XLIX, S. 457.

8. Virchows Archiv, Bd. GVII, S. 108.

9. Société médicale des Hôpiteaux (26 juillet 1889). 